\title{
Lidar Estimates of the Anisotropy of Wind Turbulence in a Stable Atmospheric Boundary Layer
}

\author{
Viktor A. Banakh * and Igor N. Smalikho \\ V.E. Zuev Institute of Atmospheric Optics SB RAS, Wave Propagation Laboratory, Tomsk 634055, Russia; \\ smalikho@iao.ru \\ * Correspondence: banakh@iao.ru; Tel.: +7-(3822)-49-29-65
}

Received: 29 June 2019; Accepted: 9 September 2019; Published: 11 September 2019

\begin{abstract}
In this paper, a method is proposed to estimate wind turbulence parameters using measurements recorded by a conically scanning coherent Doppler lidar with two different elevation angles. This methodology helps determine the anisotropy of the spatial correlation of wind velocity turbulent fluctuations. The proposed method was tested in a field experiment with a Stream Line lidar (Halo Photonics, Brockamin, Worcester, United Kingdom) under stable temperature stratification conditions in the atmospheric boundary layer. The results show that the studied anisotropy coefficient in a stable boundary layer may be up to three or larger.
\end{abstract}

Keywords: coherent Doppler lidar; wind turbulence; anisotropy; stable atmospheric boundary layer

\section{Introduction}

One of the consequences of density stratification in the atmosphere or ocean is the formation of disk-shaped density inhomogeneities due to the collapse of internal waves arising in stratified fluids [1]. In a stably stratified atmosphere, this leads to the appearance of anisotropic discoid turbulent temperature inhomogeneities. Radio occultation methods are widely used to study anisotropic turbulence in free atmosphere [2-4]. In accordance with previously published results [2,3], the spatial spectrum of the temperature turbulence in a stable atmosphere can be represented as the sum of an isotropic and anisotropic component, which are statistically independent. The results presented in [4] show that anisotropic turbulence begins to play the predominant role at heights above four to five kilometers, where the anisotropy coefficient (ratio of the horizontal scale to the vertical scale of the correlation of air temperature fluctuations) can exceed 30.

The anisotropy of turbulent fluctuations of temperature and wind velocity manifests in the atmospheric boundary layer (ABL) as well. To date, a number of questions regarding the anisotropy of wind turbulence remain unexplored. In particular, in the scientific literature, no data have been published on the anisotropy of wind turbulence in a stable ABL in the presence of low-level jets (LLJs). Typically, LLJs with maximum wind speed of $15-25 \mathrm{~m} / \mathrm{s}$ occur at night, when stratification of the air temperature is stable, at a height of 200-700 m above ground level. The mechanism of formation of the nocturnal LLJ was described in detail by Blackadar [5]. Accounting for wind turbulence anisotropy is important in the development of ABL mathematical models used for various practical applications, such as weather forecast, wind energy, air transport safety, diffusion of atmospheric impurities, etc.

In the ABL, air flow is always turbulent. Therefore, components of the wind vector $\mathbf{V}=\left\{V_{z}, V_{x}, V_{y}\right\}$ (where $V_{z}$ is the vertical component, and $V_{x}$ and $V_{y}$ are horizontal components) are random functions at time $t$ and radius vector $\mathbf{r}=\{z, x, y\}$ in a Cartesian coordinate system centered at a point on the Earth's surface ( $z$ is the vertical coordinate, $x$ and $y$ are the horizontal coordinates). Let the direction of the average horizontal wind be parallel to the axis $x$, and the average value of the vertical component be zero. In this case, $V_{x}$ is the longitudinal component and $V_{y}$ is the transverse (on the horizontal plane) 
component of the wind velocity vector. Average values $\left\langle V_{z}>\right.$ and $\left\langle V_{y}>\right.$ are equal to zero. Hereinafter, the angular brackets denote ensemble averaging. Denote the fluctuations of the vertical, longitudinal, and transversal components of the wind as $w=V_{z}, u=V_{x}-\left\langle V_{x}\right\rangle$, and $v=V_{y}$, respectively.

Pulsed coherent Doppler lidars (PCDLs) are widely applied to investigate wind turbulence in the ABL [6-25]. Strategies to conduct measurements with a scanning PCDL to study wind turbulence have been proposed [6,15], along with methods to estimate turbulence parameters, such as momentum fluxes $\langle w u\rangle,\langle w v\rangle$, and $\langle u v\rangle$; and variances of the vertical $\sigma_{w}^{2}=\left\langle w^{2}\right\rangle$, longitudinal $\sigma_{u}^{2}=<$ $u^{2}>$, and transversal $\sigma_{v}^{2}=\left\langle v^{2}>\right.$ components of wind velocity vector, respectively. These methods provide an estimation of the differences in magnitude of $\sigma_{w}^{2}, \sigma_{u}^{2}$, and $\sigma_{v}^{2}$ due to wind turbulence anisotropy, but do not allow estimation with respect to the spatial scales of the correlation of turbulent fluctuations of the wind velocity components, such as the integral spatial scales of longitudinal correlation $L_{w}=\int_{0}^{\infty} d r B_{w}(r, 0,0) / \sigma_{w}^{2}, L_{u}=\int_{0}^{\infty} d r B_{u}(0, r, 0) / \sigma_{u}^{2}$, and $L_{v}=\int_{0}^{\infty} d r B_{v}(0,0, r) / \sigma_{v}^{2}$. Here, $B_{w}(r, 0,0)=\left\langle w\left(z+r, x_{0}, y_{0}\right) w(z, x, y)>, B_{u}(0, r, 0)=\langle u(z, x+r, y) u(z, x, y)\rangle\right.$, and $B_{v}(0,0, r)=<$ $v(z, x, y+y) v(z, x, y)>$ are spatial correlation functions of the vertical, longitudinal, and transversal components of the wind velocity vector, respectively.

A method was proposed for estimation of the turbulent energy dissipation rate $\varepsilon$ and the radial velocity variance $\bar{\sigma}_{r}^{2}$ with measurements by a conically scanning PCDL at elevation angle $\phi[19,20]$. The estimate for $\bar{\sigma}_{r}^{2}$ found by the method described $[19,20]$, is a result of averaging the variances of the radial velocities measured with the lidar at all azimuth angles $\theta$, which are set during the scan. With the assumption that the turbulence structure is described by the von Kàrmàn model [26,27], the integral scale of longitudinal correlation of the radial velocity fluctuations $L_{V}$ can be calculated from the lidar estimates $\varepsilon$ and $\bar{\sigma}_{r}^{2}$ by the following equation $[19,20]$ :

$$
L_{V}=0.698\left(\bar{\sigma}_{r}^{2}\right)^{3 / 2} / \varepsilon,
$$

The integral scale $L_{V}$ is, just as $\bar{\sigma}_{r}^{2}$, the result of averaging over all the azimuth angles $\theta$. Similar to $\bar{\sigma}_{r}^{2}$, the scale $L_{V}$ at a fixed height depends on the elevation angle $\phi$ employed in the conical scanning due to the turbulence anisotropy, even at horizontally statistically homogeneous wind velocity fluctuations. If the scanning is conducted at the angle $\phi=\tan ^{-1}(1 / \sqrt{2}) \approx 35.3^{\circ}$, then the kinetic energy of turbulence $E=(1 / 2)\left(\sigma_{w}^{2}+\sigma_{u}^{2}+\sigma_{v}^{2}\right)$ can be calculated from the lidar estimates $\bar{\sigma}_{r}^{2}$ as $E=(3 / 2) \bar{\sigma}_{r}^{2}[6,19,20]$.

In this paper, a method is proposed to estimate wind turbulence parameters, including the integral spatial scales of turbulence based on Equation (1), from lidar measurements employing conical scanning at different elevation angles. This method also allows us to determine the anisotropy of the spatial correlation of wind velocity fluctuations. The method was tested in an experiment with a Stream Line lidar (Halo Photonics, Brockamin, Worcester, United Kingdom) under stable temperature stratification conditions in the atmospheric boundary layer.

\section{Lidar Method to Determine Parameters of Anisotropic Turbulence}

The instantaneous radial velocity $V_{r}$, which is the projection of the wind velocity vector onto the optical axis of the probing beam, at point $\mathbf{r}=R \mathbf{S}(\theta, \phi)$, can be represented in the form [14]:

$$
V_{r}(R, \theta, \varphi)=\mathbf{S}(\theta, \varphi) \cdot \mathbf{V}(R \mathbf{S}(\theta, \varphi))
$$

where $R$ is the distance from the lidar to the point $\mathbf{r}$ and $\mathbf{S}(\theta, \phi)=\{\sin \phi, \cos \phi \cos \theta, \cos \phi \sin \theta\}$ is the unit vector along the optical axis. Difference $V_{r}{ }^{\prime}=V_{r}-<V_{r}>$ represents turbulent fluctuations in the radial velocity. Due to statistical inhomogeneity and anisotropy, the radial velocity variance $\sigma_{r}^{2}=\left\langle\left(V_{r}{ }^{\prime}\right)^{2}\right\rangle$ is a function of both the parameters, characterizing wind turbulence, and of the distance $R$ and the angles $\theta$ and $\phi$. We assume that the wind field is statistically homogeneous in the 
horizontal plane. Then, the radial velocity variance $\bar{\sigma}_{r}^{2}(R, \varphi)=(2 \pi)^{-1} \int_{0}^{2 \pi} d \theta \sigma_{r}^{2}(R, \theta, \varphi)$ averaged over all azimuth angles $\theta$ (in the range from 0 to $2 \pi$ ) can be represented in the form [6,20]:

$$
\bar{\sigma}_{r}^{2}(h / \sin \varphi, \varphi)=(\sin \varphi)^{2} \sigma_{w}^{2}(h)+(\cos \varphi)^{2} \sigma_{H}^{2}(h),
$$

where $h=R \sin \phi=z$ is the height and $\sigma_{H}^{2}=\left(\sigma_{u}^{2}+\sigma_{v}^{2}\right) / 2$ is the wind velocity variance defined as an average of variances of the longitudinal and transversal components of the wind velocity vector in the horizontal plane. Experiments [28] showed that the variance $\sigma_{w}^{2}$ differs much more from the variances $\sigma_{u}^{2}$ and $\sigma_{v}^{2}$ than variances $\sigma_{v}^{2}$ and $\sigma_{u}^{2}$ differ from each other. Often, the variances $\sigma_{v}^{2}$ and $\sigma_{u}^{2}$ coincide. Therefore, for an experimental study of the anisotropy of wind turbulence, measuring only two variances: $\sigma_{w}^{2}$ and $\sigma_{H}^{2}=\left(\sigma_{u}^{2}+\sigma_{v}^{2}\right) / 2$ may suffice.

According to Equation (3), the variance $\bar{\sigma}_{r}^{2}$, besides the height $h$, depends on the elevation angle $\phi$ due to the anisotropy of turbulence $\left(\sigma_{w}^{2}(h) \neq \sigma_{H}^{2}(h)\right)$. To obtain estimates of the variances $\sigma_{w}^{2}$ and $\sigma_{H^{\prime}}^{2}$ simultaneous lidar measurements at two different elevation angles are necessary. Given two different angles $\phi=\phi_{1}$ and $\phi=\phi_{2}\left(\phi_{1} \neq \phi_{2}\right)$ in Equation (3), we obtain a system of equations for the height profiles of the variances $\sigma_{w}^{2}(h)$ and $\sigma_{H}^{2}(h)$ :

$$
\left\{\begin{array}{l}
\bar{\sigma}_{r}^{2}\left(h / \sin \varphi_{1}, \varphi_{1}\right)=\left(\sin \varphi_{1}\right)^{2} \sigma_{w}^{2}(h)+\left(\cos \varphi_{1}\right)^{2} \sigma_{H}^{2}(h) \\
\bar{\sigma}_{r}^{2}\left(h / \sin \varphi_{2}, \varphi_{2}\right)=\left(\sin \varphi_{2}\right)^{2} \sigma_{w}^{2}(h)+\left(\cos \varphi_{2}\right)^{2} \sigma_{H}^{2}(h)
\end{array},\right.
$$

To retrieve the vertical profile of the turbulent kinetic energy $E(h)$ from lidar data, we take $\phi_{1}=35.3^{\circ}$ in Equation (4) [6]. The angle $\phi_{2}$ is taken as $60^{\circ}$. Then, the solution of the system of Equation (4) has the following form:

$$
\begin{aligned}
& \sigma_{w}^{2}(h)=\frac{1}{5}\left[8 \bar{\sigma}_{r}^{2}\left(h / \sin \varphi_{2}, \varphi_{2}\right)-3 \bar{\sigma}_{r}^{2}\left(h / \sin \varphi_{1}, \varphi_{1}\right)\right], \\
& \sigma_{H}^{2}(h)=\frac{1}{5}\left[9 \bar{\sigma}_{r}^{2}\left(h / \sin \varphi_{1}, \varphi_{1}\right)-4 \bar{\sigma}_{r}^{2}\left(h / \sin \varphi_{2}, \varphi_{2}\right)\right],
\end{aligned}
$$

where $\phi_{1}=35.3^{\circ}$ and $\phi_{2}=60^{\circ}$.

The turbulent energy dissipation rate $\varepsilon$ is a characteristic of locally isotropic wind turbulence within the inertial subrange. In the case of statistical homogeneity of the wind field in the horizontal plane, the dissipation rate is a function of height $h$ and does not depend on horizontal coordinates $x$ and $y$. After substitution of the values of the variance $\bar{\sigma}_{r}^{2}\left(h / \sin \varphi_{i}, \varphi_{i}\right)$ and the dissipation rate $\varepsilon(h)$ obtained from lidar measurements at different elevation angles $\phi_{i}(i=1,2)$ into Equation (1), the integral scales $L_{V}\left(h, \phi_{i}\right)$ at the angles $\phi_{i}$ are calculated. The estimation method of $\bar{\sigma}_{r}^{2}$ and $\varepsilon$ from the data measured by the conically scanning PCDL is described in [20]. Employing the von Kàrmàn model in Equation (1) yields the vertical profiles of longitudinal correlation scales of fluctuations of the vertical $L_{w}(h)$ and horizontal $L_{H}(h)$ wind velocity, if the variance $\bar{\sigma}_{r}^{2}$ in Equation (1) is replaced with $\sigma_{w}^{2}(h)$ and $\sigma_{H}^{2}(h)$, respectively. Because $\sigma_{H}^{2}=\left(\sigma_{u}^{2}+\sigma_{v}^{2}\right) / 2$, we assume that the value of $L_{H}$ is between values of $L_{u}$ and $L_{v}$.

On obtaining scales $L_{V 1}=L_{V}\left(h, \phi_{1}\right)$ and $L_{V 2}=L_{V}\left(h, \phi_{2}\right)$ from lidar measurements at the scanning elevation angles $\phi_{1}=35.3^{\circ}$ and $\phi_{2}=60^{\circ}$, and employing Equations (1), (5), and (6), the scales $L_{w}$ and $L_{H}$ can be respectively calculated as:

$$
\begin{aligned}
& L_{w}=\left(\frac{8 L_{V 2}^{2 / 3}-3 L_{V 1}^{2 / 3}}{5}\right)^{3 / 2}, \\
& L_{H}=\left(\frac{9 L_{V 1}^{2 / 3}-4 L_{V 2}^{2 / 3}}{5}\right)^{3 / 2} .
\end{aligned}
$$


To apply the described method to estimate the variances and correlation scales of fluctuations of vertical and horizontal wind velocity, measurements should be recorded simultaneously by two lidars scanning at different elevation angles. However, considering that the lidar data has to be averaged over a long period of time to obtain a statistically significant estimate of the variance of radial velocity, this method can be applied using a single lidar.

Therefore, as shown in Figure 1, the lidar probing beam conducts conical scanning about the vertical axis alternatively at the elevation angles of $35.3^{\circ}$ (odd scan numbers) and $60^{\circ}$ (even scan numbers).

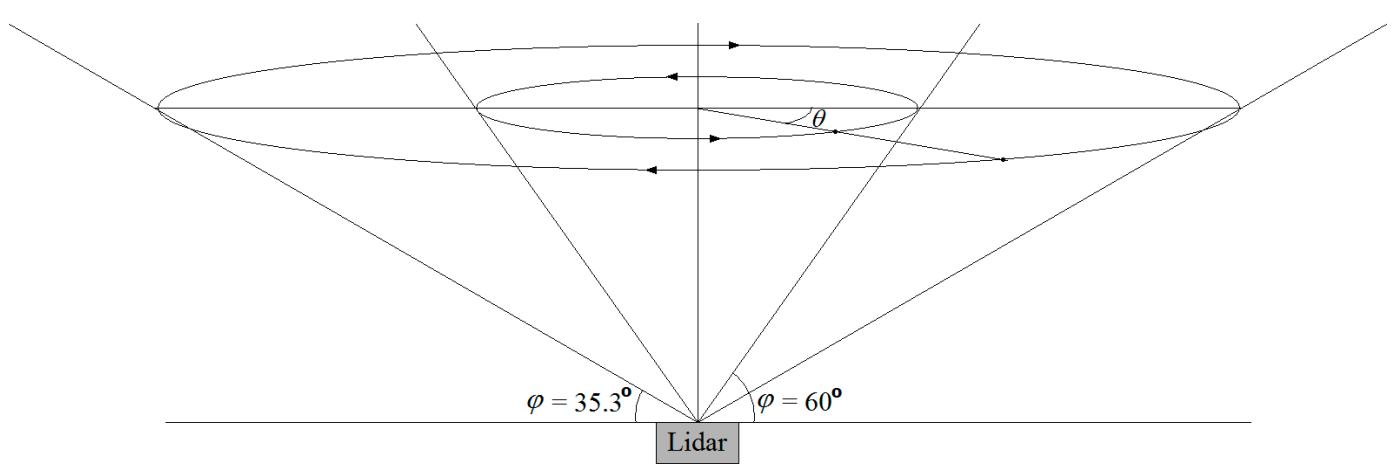

Figure 1. Geometry of lidar measurement employing alternate conical scanning around the vertical axis at the elevation angles $(\phi)$ of $35.3^{\circ}$ and $60^{\circ}$.

Before the measurements were taken, elevation angle was set to $\phi=35.3^{\circ}$. Then, the scanning starts with constant angular velocity $\omega_{s}=d \theta / d t$. During the scanning, the azimuth angle $\theta$ of the probing direction varies from $0^{\circ}$ to $360^{\circ}$. After a full turn, the scanning stops and the elevation angle of $35.3^{\circ}$ changes to $60^{\circ}$, which takes approximately $1 \mathrm{~s}$. Then, the scanning starts in the opposite direction. The azimuth angle $\theta$ changes from $360^{\circ}$ to $0^{\circ}$. At $\theta=0^{\circ}$, the elevation angle of $60^{\circ}$ again changes to $35.3^{\circ}$ and the procedure repeats.

\section{Experiment}

The experiment was conducted on 6-24 June 2018 at the Basic Experimental Observatory (BEO) of the Institute of Atmospheric Optics SB RAS (IAO) in Tomsk, Russia (56.475448 ${ }^{\circ} \mathrm{N}, 85.048115^{\circ}$ E), employing a Stream Line PCDL (Halo Photonics, Brockamin, Worcester, United Kingdom). The experimental geometry is shown in Figure 1, which shows conical scanning with alternating elevation angle $\left(35.3^{\circ}\right.$ and $\left.60^{\circ}\right)$. The duration of every scan was $T_{\text {scan }}=60 \mathrm{~s}$. Adding the time $\delta t$ $\approx 1 \mathrm{~s}$ needed to change the elevation angle from $35.3^{\circ}$ to $60^{\circ}$ and vice versa, the duration of one measurement cycle $T_{\text {circl }}=2\left(T_{\text {scan }}+\delta t\right)$ was a little bit longer than $2 \mathrm{~min}$. For accumulation of raw lidar data, $N_{a}=7500$ laser shots were used. The pulse repetition frequency was $f_{p}=15 \mathrm{kHz}$. Thus, the duration of measurements for every azimuth angle $\Delta t=N_{a} / f_{p}=0.5 \mathrm{~s}$. The azimuth resolution was $\Delta \theta=360^{\circ} / M=3^{\circ}$, where $M=T_{\text {scan }} / \Delta t=120$ was the number of rays for one conical scan.

As a result of the measurements, we obtained arrays of estimates of the signal-to-noise ratio SNR $\left(R_{k}, \theta_{m} ; n\right)$ and the radial velocity $V_{L}\left(R_{k}, \theta_{m} ; n\right)$. Here, the SNR is the ratio of average heterodyne signal power to the average detector noise power in a $50 \mathrm{MHz}$ bandwidth. Estimates of SNR and $V_{L}$ are functions of the parameters $R_{k}, \theta_{m}$, and $n$, where $R_{k}=R_{0}+k \Delta R$ is the distance from the lidar to the center of the sensing volume, $k=0,1,2, \ldots, K-1 ; \Delta R=18 \mathrm{~m}$ is the range gate length; $\theta_{m}=m \Delta \theta$ is the dependence of the azimuth angle on the ray number $m=0,1,2, \ldots, M-1$ at the elevation angle $\phi=35.3^{\circ}$; $\theta_{m}=360^{\circ}-m \Delta \theta$ is the azimuth angle at $\phi=60^{\circ} ; n=1,3,5,7, \ldots$ is the conical scan number at the elevation angle $\phi=35.3^{\circ}$; and $n=2,4,6,8, \ldots$ is the conical scan number at the elevation angle $\phi=60^{\circ}$. Each of the arrays SNR $\left(R_{k}, \theta_{m} ; n\right)$ and $V_{L}\left(R_{k}, \theta_{m} ; n\right)$ is divided into two sub-arrays: $\operatorname{SNR}_{i}\left(R_{k}, \theta_{m} ; n\right)$ 
and $V_{L i}\left(R_{k}, \theta_{m} ; n\right)$ with odd $(i=1)$ and even $(i=2)$ numbers $n$. The subscript $i=1$ corresponds to the elevation angle $\phi=35.3^{\circ}$, whereas $i=2$ denotes $\phi=60^{\circ}$.

To increase the accuracy of SNR measurement, SNR estimates were averaged over all azimuth angles for every distance $R_{k}$ and scan number $n, \operatorname{SN}_{i}\left(R_{k}, n\right)=M^{-1} \sum_{m=0}^{M-1} \operatorname{SNR}_{i}\left(R_{k}, \theta_{m} ; n\right)$. Estimates of the wind velocity vector $V_{i}\left(R_{k}, n\right)=\left\{V_{z i}\left(R_{k}, n\right), V_{x i}\left(R_{k}, n\right), V_{y i}\left(R_{k}, n\right)\right\}$, where $V_{z i}$ is the vertical component and $V_{x i}$ and $V_{y i}$ are the horizontal components, were determined from the arrays of lidar estimates of the radial velocity $V_{L i}\left(R_{k}, \theta_{m} ; n\right)$ using the direct sine-wave fitting method [14,29]. The wind speed $U_{i}$ and the wind direction angle $\theta_{V i}$ were calculated from the horizontal components of the velocity vector as $U_{i}=\left|V_{x i}+j V_{y i}\right|$ and $\theta_{V i}=\arg \left\{V_{x i}+j V_{y i}\right\}$, respectively, where $j$ is an imaginary unit.

\section{Results of the Measurements}

To retrieve information about turbulence from lidar data, the probability of bad (false) estimates of radial velocity has to be nearly zero [30]. That is, the SNR during the measurements should be high. For accumulation number $N_{a}=7500$, the SNR should exceed $-16 \mathrm{~dB}$. Therefore, to study the anisotropy of wind turbulence in a stable $\mathrm{ABL}$, we had to select data corresponding to the stable temperature stratification and SNR $>-16 \mathrm{~dB}$. Lidar data obtained in the 12-hour period from 20:00 on $23 \mathrm{July}$ to 08:00 on 24 July met this requirement. Using the data for the average temperature of air $T(h, t)$ measured by sonic anemometers during this time interval at heights $h_{1}=3 \mathrm{~m}$ and $h_{1}=42 \mathrm{~m}$, we calculated the potential temperature derivative $d T_{p} / d h$ by the equation: $d T_{p} / d h=\left[T\left(h_{2}, t\right)-T\left(h_{1}, t\right)\right] /\left(h_{2}-h_{1}\right)+y_{a}$, where $y_{a}=0.0098^{\circ} \mathrm{K} / \mathrm{m}$ is the adiabatic gradient of dry air temperature. Under the condition $d T_{p} / d h>0$, the temperature stratification is stable; if $d T_{p} / d h=0$, the stratification is neutral; and when $d T_{p} / d h<0$, the stratification is unstable. Calculations show that beginning with 20:00 on 23 July to 08:00 on 24 July, the value of $d T_{p} / d h$ varied in the range of 0.005 to $0.02{ }^{\circ} \mathrm{K} / \mathrm{m}$. Therefore, the temperature stratification of the surface atmospheric layer was stable in this period.

The estimates of the radial velocity with subscript $k$ obtained from lidar measurements at different values of the elevation angles, $\phi_{1}=35.3^{\circ}$ and $\phi_{2}=60^{\circ}$, correspond to different heights, $h_{k i}=h_{0 i}+k \Delta h_{i}$, where $h_{0 i}=R_{0} \sin \phi_{i}$ is the initial height and $\Delta h_{i}=\Delta R \sin \phi_{i}$ is the vertical step. By sorting the obtained data in heights, two two-dimensional (2D) composite plots corresponding to the elevation angles of $35.3^{\circ}$ and $60^{\circ}$ were sampled for each of the following parameters: the signal-to-noise ratio $\mathrm{S} \overline{\mathrm{N}} \mathrm{R}_{i}\left(h_{k i}, t_{n}\right)$, wind speed $U_{i}\left(h_{k i}, t_{n}\right)$, wind direction angle $\theta_{V i}\left(h_{k i}, t_{n}\right)$, and the vertical wind speed $V_{z i}\left(h_{k i}, t_{n}\right)$. Here, $t_{n}$ $=t_{0}+n T_{\text {circl }} / 2$ is the current time and $t_{0}$ is the measurement starting time. The obtained distributions are shown in Figure 2, which shows that the $\mathrm{SN}_{1}\left(h, t_{n}\right)$ for the elevation angle of $35.3^{\circ}$ becomes much smaller than $\mathrm{SNR}_{2}\left(h, t_{n}\right)$ for the elevation angle of $60^{\circ}$ starting from heights at $400-500 \mathrm{~m}$. This is caused by the longer distance to the probed volume at $\phi=35.3^{\circ}$ than at $\phi=60^{\circ}$ and, possibly, by the horizontal inhomogeneity of the aerosol backscatter coefficient. In the zones where $\mathrm{S} \bar{N}_{i}\left(h_{k i}, t_{n}\right)>-16$ $\mathrm{dB}$, differences in the height-time distributions of the wind speeds $\mathrm{U}_{1}\left(h, t_{n}\right)$ and $U_{2}\left(h, t_{n}\right)$ and the wind direction angles $\theta_{V 1}\left(h, t_{n}\right)$ and $\theta_{V 2}\left(h, t_{n}\right)$ are not as significant. (Compare Figure $2 \mathrm{c}$ with Figure $2 \mathrm{~d}$ and Figure 2e with Figure 2f.) These differences are mainly caused by turbulent variations in the wind field. The low-level jet (LLJ) at heights of 350-700 $\mathrm{m}$ is easily observed. At the LLJ center, the wind speed sometimes achieved $25 \mathrm{~m} / \mathrm{s}$. The estimates of the vertical component of the wind velocity vector at $\mathrm{SNR}_{i}\left(h_{k i}, t_{n}\right)>-16 \mathrm{~dB}$ do not go beyond, with one exception-the range from -1 to $1 \mathrm{~m} / \mathrm{s}$ (Figure $2 \mathrm{~g}-\mathrm{h}$ ). This agrees with the results reported by Lolli et al. [31]. Due to large-scale turbulent inhomogeneities of the wind flow and fast mesoscale processes, a significant difference was observed in estimates of vertical wind velocity $V_{z 1}\left(h, t_{n}\right)$ and $V_{z 2}\left(h, t_{n}\right)$.

To obtain estimates for the wind turbulence parameters, we used the measured data obtained at $\mathrm{S} \overline{\mathrm{N}} \mathrm{R}_{i}\left(h_{k i}, t_{n}\right)>-16 \mathrm{~dB}$, when the probability of bad estimates of the radial velocity [32] was close to zero. According to the data in Figure 2a-b, this requirement was fulfilled for heights up to $600 \mathrm{~m}$. The turbulent energy dissipation rates $\varepsilon_{1}\left(h_{k 1}, t_{n}\right)$ and $\varepsilon_{2}\left(h_{k 2}, t_{n}\right)$, radial velocity variances $\sigma_{r 1}^{2}\left(h_{k 1}, t_{n}\right)$ and $\sigma_{r 2}^{2}\left(h_{k 2}, t_{n}\right)$, and integral scales of turbulence $L_{V 1}\left(h_{k 1}, t_{n}\right)$ and $L_{V 2}\left(h_{k 2}, t_{n}\right)$ were estimated from 
the arrays of fluctuations of lidar estimates of the radial velocity $V_{L i}{ }^{\prime}$ using the method described thoroughly by Smalikho and Banakh [20]. Here, $V_{L i}{ }^{\prime}\left(R_{k}, \theta_{m} ; n\right)=V_{L i}\left(R_{k}, \theta_{m} ; n\right)-\mathbf{S}_{i}\left(\theta_{m}\right) \cdot \mathbf{V}\left(R_{k}, n\right) ; \mathbf{S}_{i}\left(\theta_{m}\right)$ $=\left\{\sin \phi_{i}, \cos \phi_{i} \cos \theta_{m}, \cos \phi_{i} \sin \theta_{m}\right\}, i=1,2 ;$ and $i=1$ and $i=2$ correspond to measurements at the elevation angles of $35.3^{\circ}$ (odd scan numbers $n$ ) and $60^{\circ}$ (even $n$ ), respectively. For the averaging, we used estimates of $V_{L i}{ }^{\prime}\left(R_{k}, \theta_{m} ; n\right)$ obtained from lidar measurements for 20 scans for each elevation angle. This corresponds to 40-min time averaging.
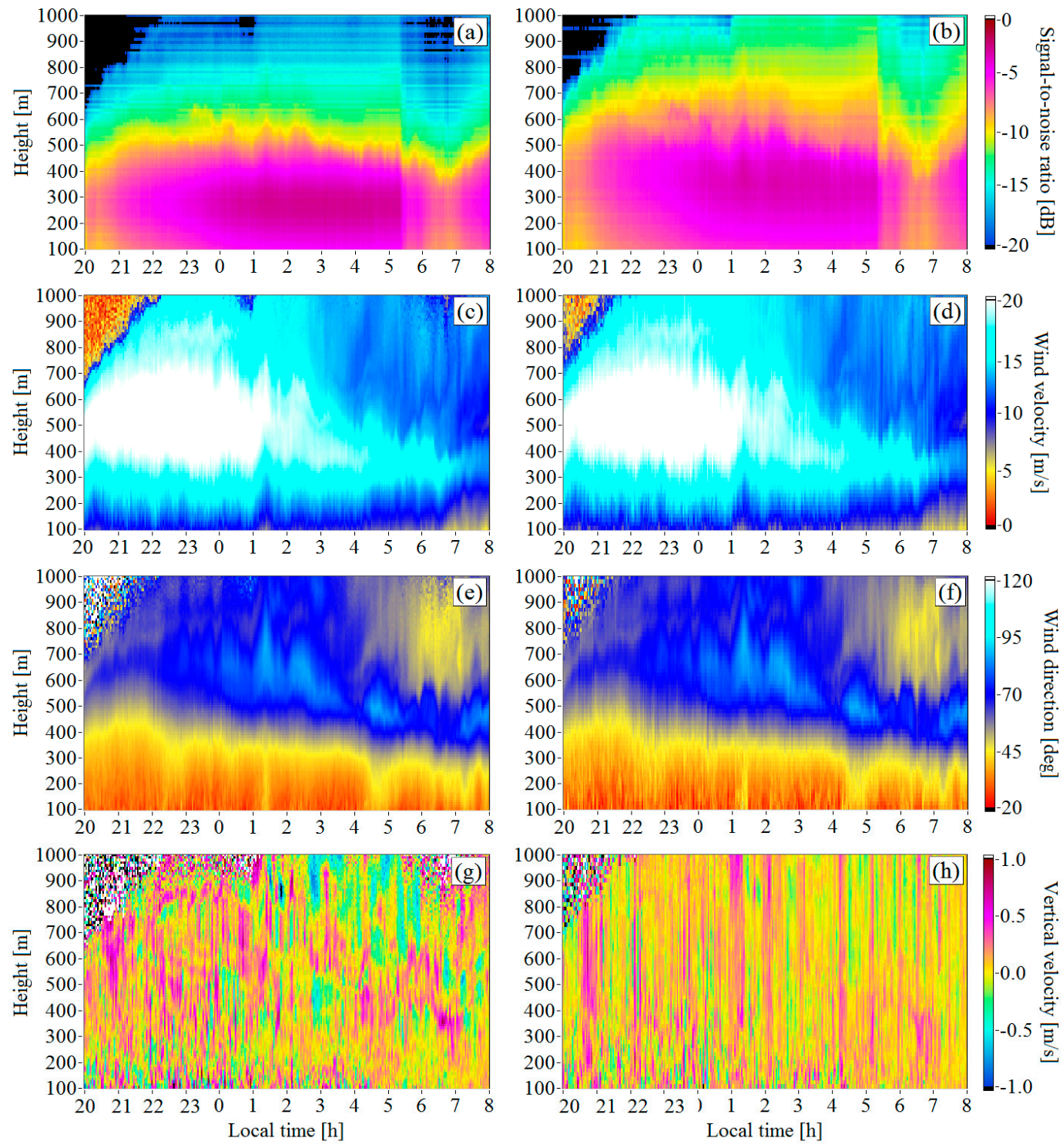

Figure 2. Height and time distribution of the $(\mathbf{a}, \mathbf{b})$ signal-to-noise ratio (SNR), (c,d) horizontal wind velocity, (e,f) wind direction angle, and $(\mathbf{g}, \mathbf{h})$ the vertical component of the wind velocity vector obtained from lidar measurements at the Basic Experimental Observatory of the Institute of Atmospheric Optics, Siberian Branch of the Russian Academy of Sciences (Tomsk, Russia) on 23-24 July 2018 employing conical scanning at elevation angles of $(\mathbf{a}, \mathbf{c}, \mathbf{e}, \mathbf{g}) 35.3^{\circ}$ and $(\mathbf{b}, \mathbf{d}, \mathbf{f}, \mathbf{h}) 60^{\circ}$.

Figure 3 depicts the obtained spatiotemporal distributions of turbulence parameters $\sigma_{r i}^{2}\left(h_{k i}, t_{n}\right)$, $\varepsilon_{i}\left(h_{k i}, t_{n}\right)$, and $L_{V i}\left(h_{k i}, t_{n}\right)$. In every distribution, the black curve shows the temporal profile of the height of maximal wind speed assessed from the data in Figure 2c. This curve is a boundary between the top and bottom parts of the LLJ and can be considered as its center. The wind turbulence is very weak in the top part of the LLJ; in particular, the turbulent energy dissipation rate does not exceed $10^{-4} \mathrm{~m}^{2} / \mathrm{s}^{3}$, and the variance of radial velocity $\sigma_{r 2}^{2}\left(h_{k 2}, t_{n}\right)$ at the elevation angle of $60^{\circ}$ is below $0.08 \mathrm{~m}^{2} / \mathrm{s}^{2}$. 

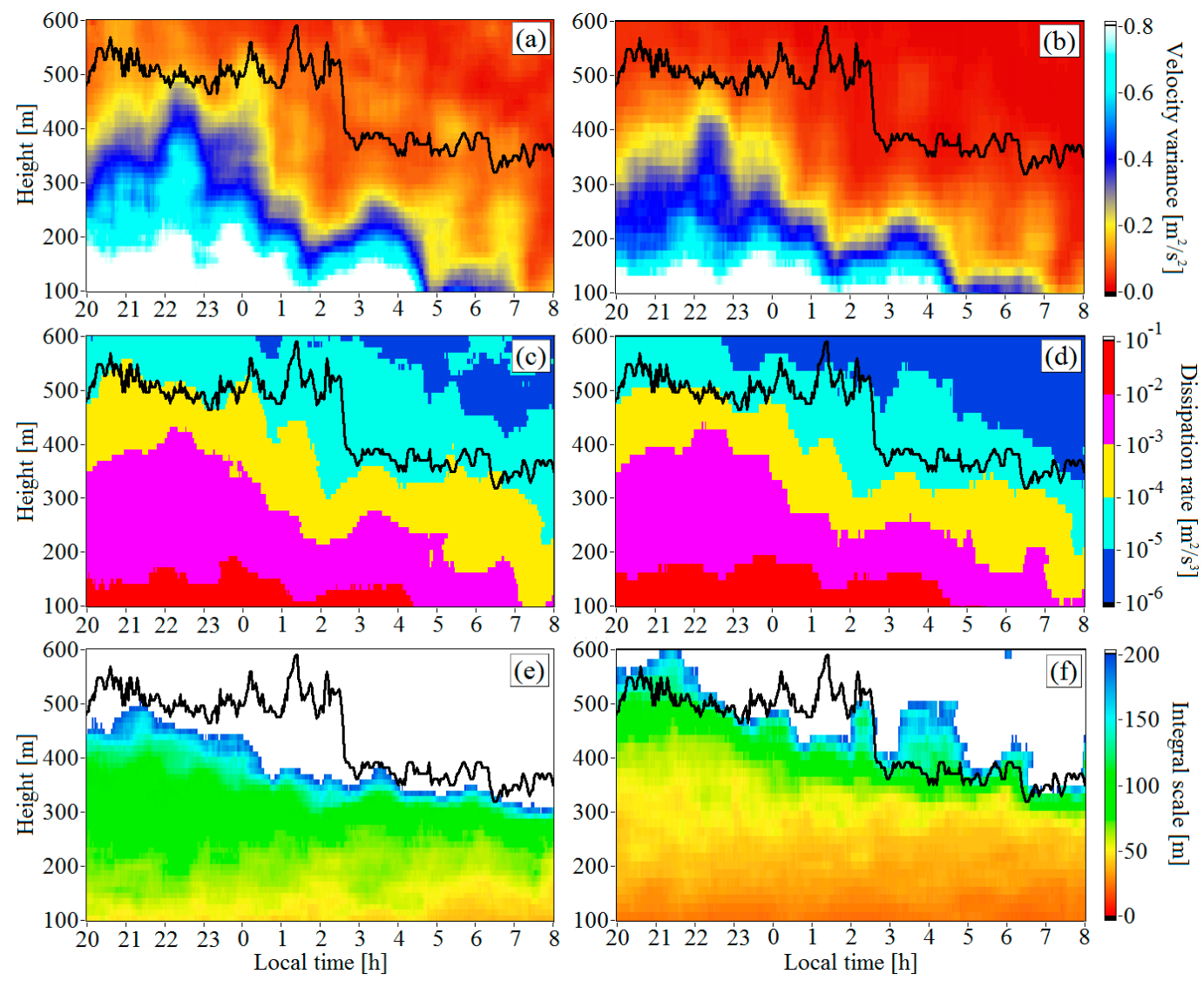

Figure 3. Height and time distributions of the $(\mathbf{a}, \mathbf{b})$ radial velocity variance, $(\mathbf{c}, \mathbf{d})$ turbulent energy dissipation rate, and $(\mathbf{e}, \mathbf{f})$ integral scale of turbulence obtained from lidar measurements at the Basic Experimental Observatory of the IAO on 23-24 July 2018 employing conical scanning at elevation angles of $(\mathbf{a}, \mathbf{c}, \mathbf{e}) 35.3^{\circ}$ and $(\mathbf{b}, \mathbf{d}, \mathbf{f}) 60^{\circ}$. Black curves show the time profile of the height of maximal wind speed in the vertical distribution of the speed within low-level jet (LLJ).

Assuming horizontal statistical homogeneity of the wind, the kinetic energy of turbulence was calculated as $E\left(h_{k 1}, t_{n}\right)=(3 / 2) \sigma_{r 1}^{2}\left(h_{k 1}, t_{n}\right)$ from the data for the variance of radial velocity $\sigma_{r 1}^{2}\left(h_{k 1}, t_{n}\right)$ obtained from measurements at the elevation angle of $35.3^{\circ}$. Combining calculated $E\left(h_{k 1}, t_{n}\right)$ and data of Figure 2c,e, and Figure 3a,c, we drew vertical profiles of the wind speed and direction, kinetic energy of turbulence, and its dissipation rate in Figure 4 . The turbulence was strong on 23 July in the bottom part of the LLJ at heights $200-400 \mathrm{~m}$ at wind speeds exceeding $15 \mathrm{~m} / \mathrm{s}$. The kinetic energy $E$ ranged from 1.0 to $0.5 \mathrm{~m}^{2} / \mathrm{s}^{2}$, and the dissipation rate varied within $710^{-3}-10^{-3} \mathrm{~m}^{2} / \mathrm{s}^{3}$. We calculated the errors of estimation of the kinetic energy $E$ and the dissipation rate $\varepsilon$ using the technique described in [30]. According to our calculations, the relative error of the kinetic energy estimates was $7 \%-10 \%$ in the bottom part of the LLJ, whereas the accuracy of the dissipation rate estimates was $6 \%-8 \%$.

In this experiment, the minimum height for retrieving vertical profiles of velocity from lidar measurements with acceptable accuracy was $100 \mathrm{~m}$. The maximum height at which measurements were recorded with sonic anemometers was $42 \mathrm{~m}$ (a sonic anemometer was installed on top of the mast, located $150 \mathrm{~m}$ from the lidar). In Figure 4, in addition to lidar data, we depict the results of measurements with the sonic anemometer at a height of $42 \mathrm{~m}$. Sonic anemometer measurement data show that, despite the conditions of stable temperature stratification, in the surface layer of the atmosphere (layer thickness of about $100 \mathrm{~m}$ in height above the ground), wind turbulence was quite strong. 

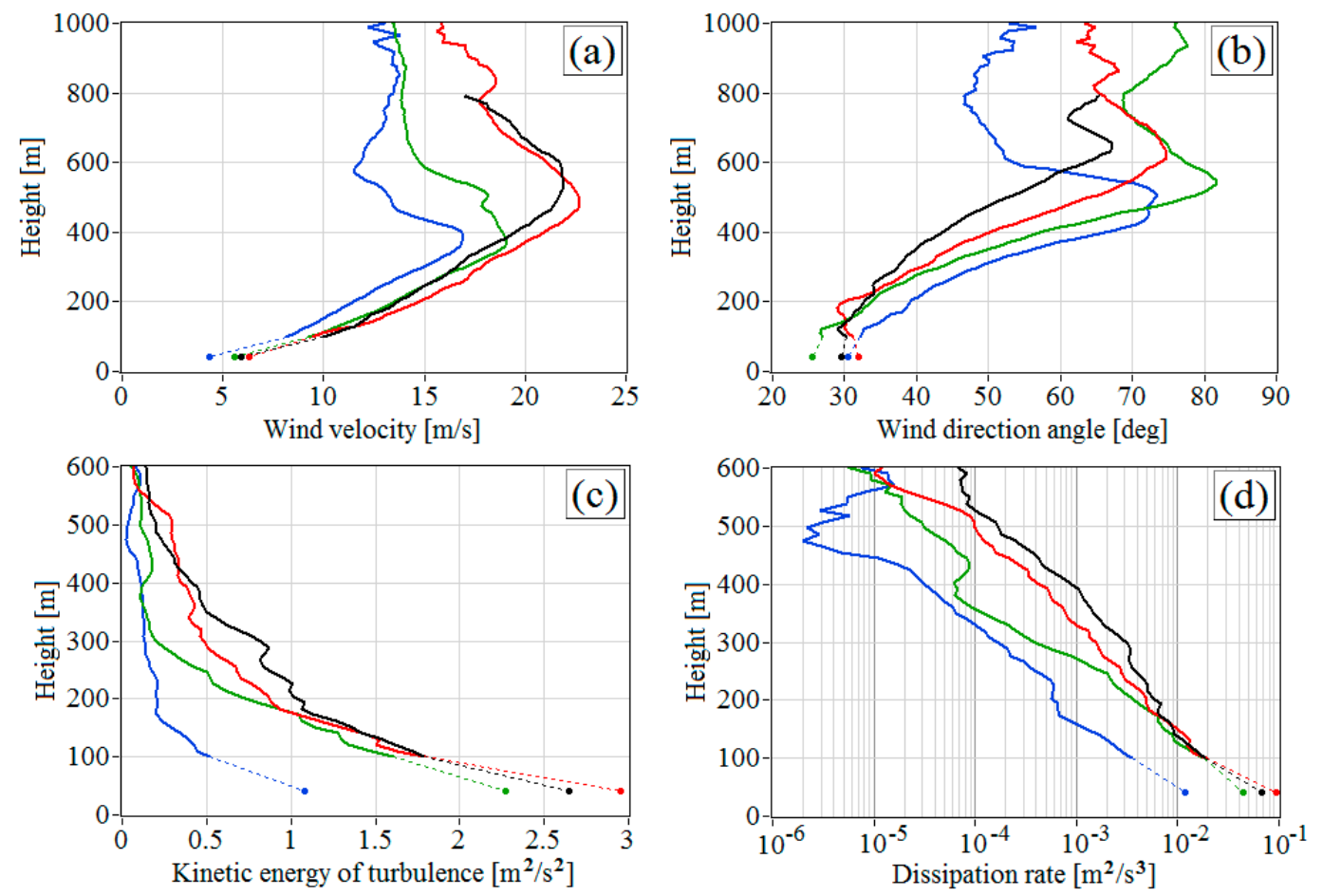

Figure 4. Vertical profiles of (a) wind velocity, (b) wind direction angle, (c) kinetic energy of turbulence, and (d) turbulent energy dissipation rate retrieved from measurements by the lidar employing conical scanning at the elevation angle of $35.3^{\circ}$ at 21:00 local time (LT) on 23 July (black curves) and 00:00 (red curves), 03:00 (green curves), and 06:00 LT (blue curves) on 24 July 2018. The profiles of wind velocity and wind direction angle were retrieved from the data of one scan (measurement duration of $1 \mathrm{~min}$ ), whereas the profiles of wind turbulence parameters were retrieved from the data of 20 scans. The circles indicate the results of measurements with a sonic anemometer at a height of $42 \mathrm{~m}$. The dotted lines "stitch" the corresponding data, which is measured using a sonic anemometer and lidar.

The turbulent energy dissipation rate is a characteristic of the locally isotropic field of wind velocities. Assuming that the wind velocity field is statistically homogeneous in the horizontal plane, the lidar estimates of the dissipation rates $\varepsilon_{1}\left(h, t_{h}\right)$ and $\varepsilon_{2}\left(h, t_{h}\right)$ obtained for the same height $h$, but for different elevation angles $\left(35.5^{\circ}\right.$ and $\left.60^{\circ}\right)$, should fully coincide on average. To verify this, we used the data in Figure $3 \mathrm{c}-\mathrm{d}$ and their vertical interpolation with a step of $10 \mathrm{~m}$, starting from $100 \mathrm{~m}$ and ending at a height of $500 \mathrm{~m}$.

Figure 5 depicts the results of the comparison of the lidar estimates of the dissipation rate $\varepsilon_{1}\left(h, t_{n}\right)$ and $\varepsilon_{2}\left(h, t_{n}\right)$ in four vertical layers: (1) 100-200, (2) 200-300, (3) 300-400, and (4) 400-500 m. The analysis of these results showed that, on average, $\varepsilon_{1}$ is $20 \%$ less than $\varepsilon_{2}$ in the first layer, $\varepsilon_{1}$ and $\varepsilon_{2}$ practically coincide in the second layer, and $\varepsilon_{1}$ exceeds $\varepsilon_{2} 1.5$ and 2 times in the third and fourth layers, respectively. The reason for the small discrepancy in dissipation rate estimates in the lowest layers is unclear. On average, at heights from $170 \mathrm{~m}$ to $320 \mathrm{~m}$, the absolute value of the discrepancy between of the dissipation rate estimates $\left[2\left(\varepsilon_{1}-\varepsilon_{2}\right) /\left(\varepsilon_{1}+\varepsilon_{2}\right)\right] \times 100 \%$ does not exceed $10 \%$. This indicates the horizontal statistical homogeneity of the wind velocity field at these heights. The significant discrepancy of the dissipation rate estimates from lidar measurements at the elevation angles of $35.3^{\circ}$ and $60^{\circ}$ in the upper layers may originate from horizontal mesoscale inhomogeneities in the wind field caused, for example, by internal atmospheric waves being stronger against the weakly turbulent background. 

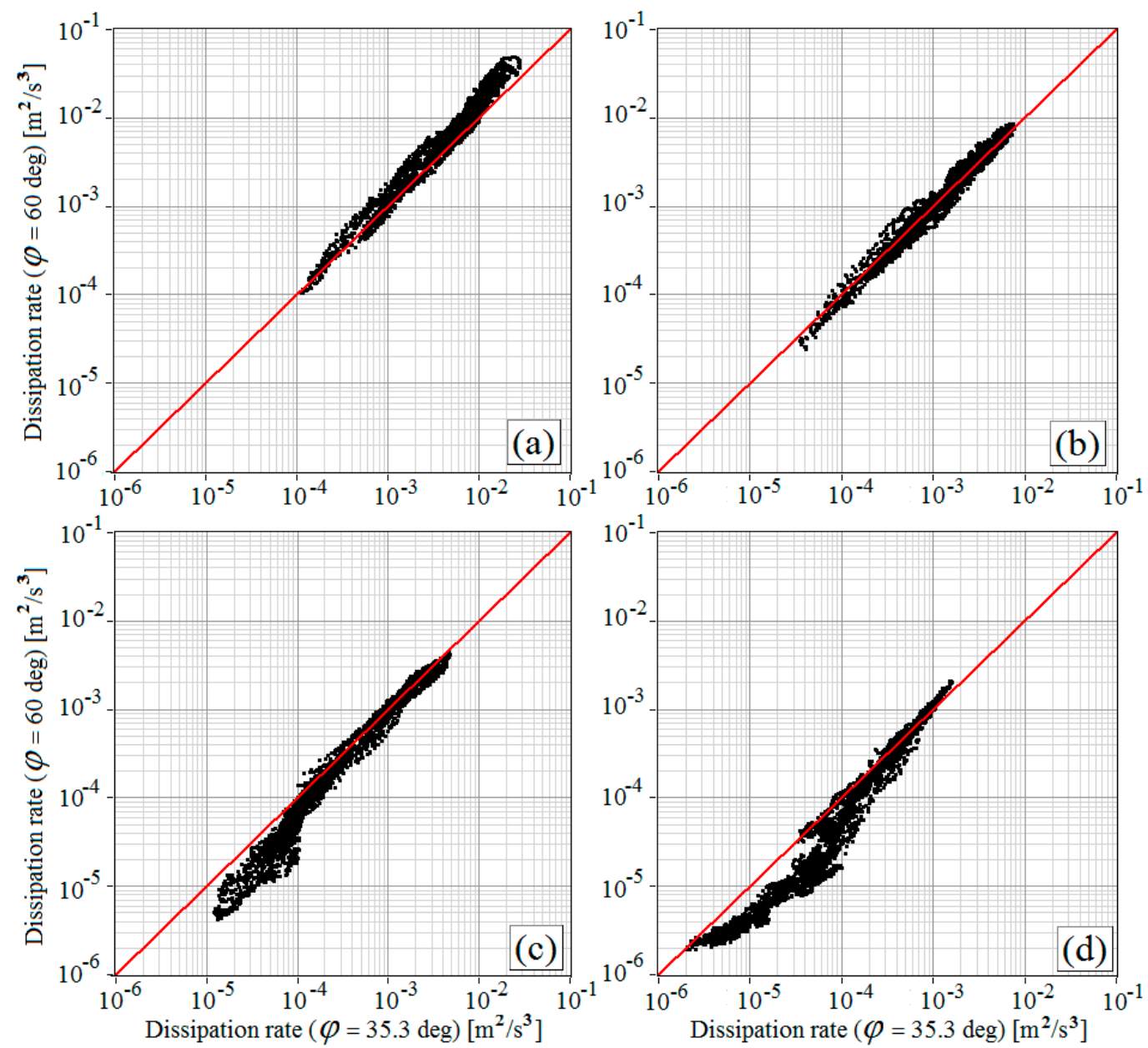

Figure 5. Comparison of estimates of the turbulent energy dissipation rate (black dots) obtained from measurements by the lidar at elevation angles of $35.3^{\circ}$ and $60^{\circ}$ from 20:00 LT on 23 July to 08:00 LT on 24 July 2018 in the vertical layers of (a) 100-200, (b) 200-300, (c) 300-400, and (d) 400-500 m. Each estimate of the dissipation rate was obtained from data of 20 conical scans.

Accessible experimental data (see, for example, [28]) indicate that in the atmospheric boundary layer, the variance of horizontal wind velocity $\sigma_{H}^{2}(h)$ exceeds the variance of the vertical component $\sigma_{w}^{2}(h)$, and the correlation scale of the fluctuations of the horizontal velocity $L_{u}(h)$ is larger than the correlation scale of fluctuations of the vertical velocity $L_{W}(h)$ due to anisotropy of wind turbulence. Since $\sigma_{H}^{2}>\sigma_{w}^{2}$, according to Equation (4), we expect that the variance $\bar{\sigma}_{r}^{2}\left(h / \sin \varphi_{1}, \varphi_{1}\right)$ exceeds $\bar{\sigma}_{r}^{2}\left(h / \sin \varphi_{2}, \varphi_{2}\right)$ in our experiment at the elevation angles $\phi_{1}=35.3^{\circ}<\phi_{2}=60^{\circ}$.

Figure 6 shows the vertical profiles of the variances $\bar{\sigma}_{r 1}^{2}\left(h_{k 1}\right)=\bar{\sigma}_{r}^{2}\left(R_{k}, \varphi_{1}\right)$ and $\bar{\sigma}_{r 2}^{2}\left(h_{k 2}\right)=\bar{\sigma}_{r}^{2}\left(R_{k}, \varphi_{2}\right)$, and integral correlation scales $L_{V 1}\left(h_{k 1}\right)=L_{V}\left(R_{k}, \phi_{1}\right)$ and $L_{V 2}\left(h_{k 2}\right)=L_{V}\left(R_{k}, \phi_{2}\right)$ of the radial velocity fluctuations, as retrieved from the lidar measurements at elevation angles $\phi=\phi_{1}=35.3^{\circ}$ and $\phi=\phi_{2}$ $=60^{\circ}$, respectively. At the same height $h$, the variance satisfies $\bar{\sigma}_{r 1}^{2}>\bar{\sigma}_{r 2}^{2}$ (Figure 6a,c,e). The integral scales $L_{V i}$ were calculated, according to Equation (1), as:

$$
L_{V i}=0.698\left(\bar{\sigma}_{r i}^{2}\right)^{3 / 2} / \varepsilon_{i}
$$



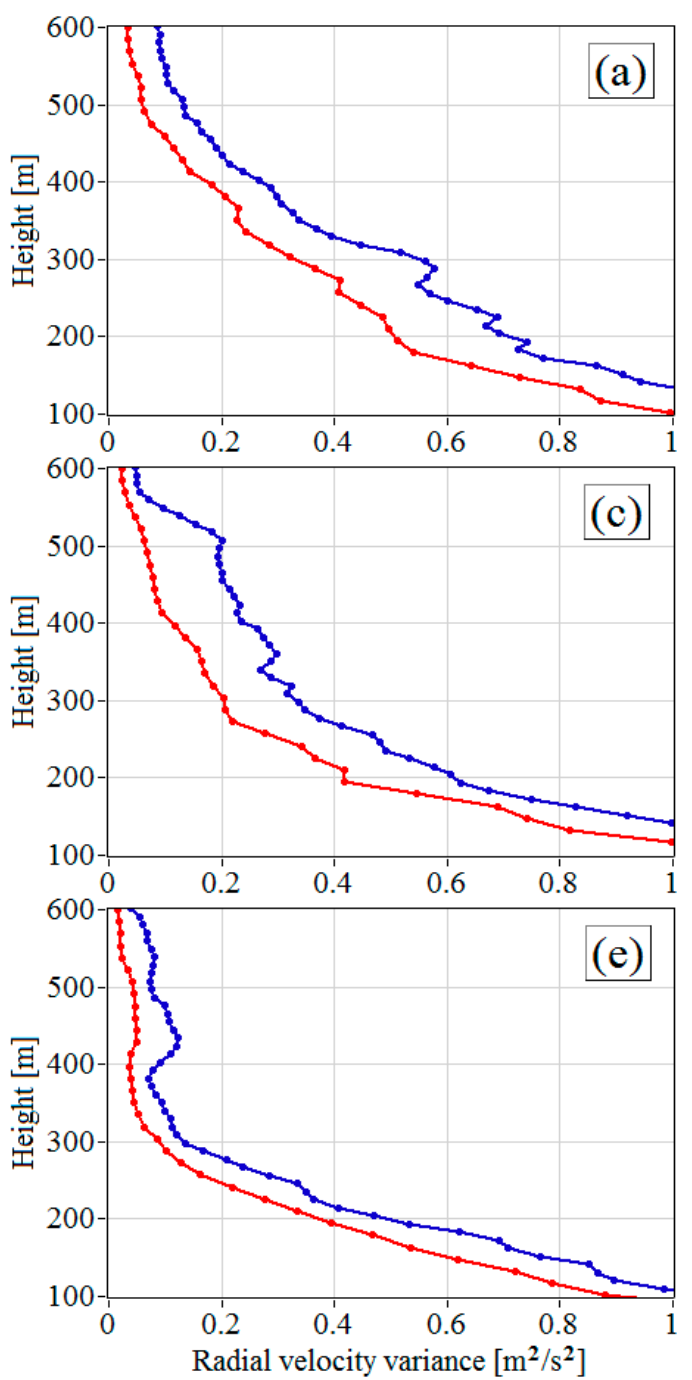
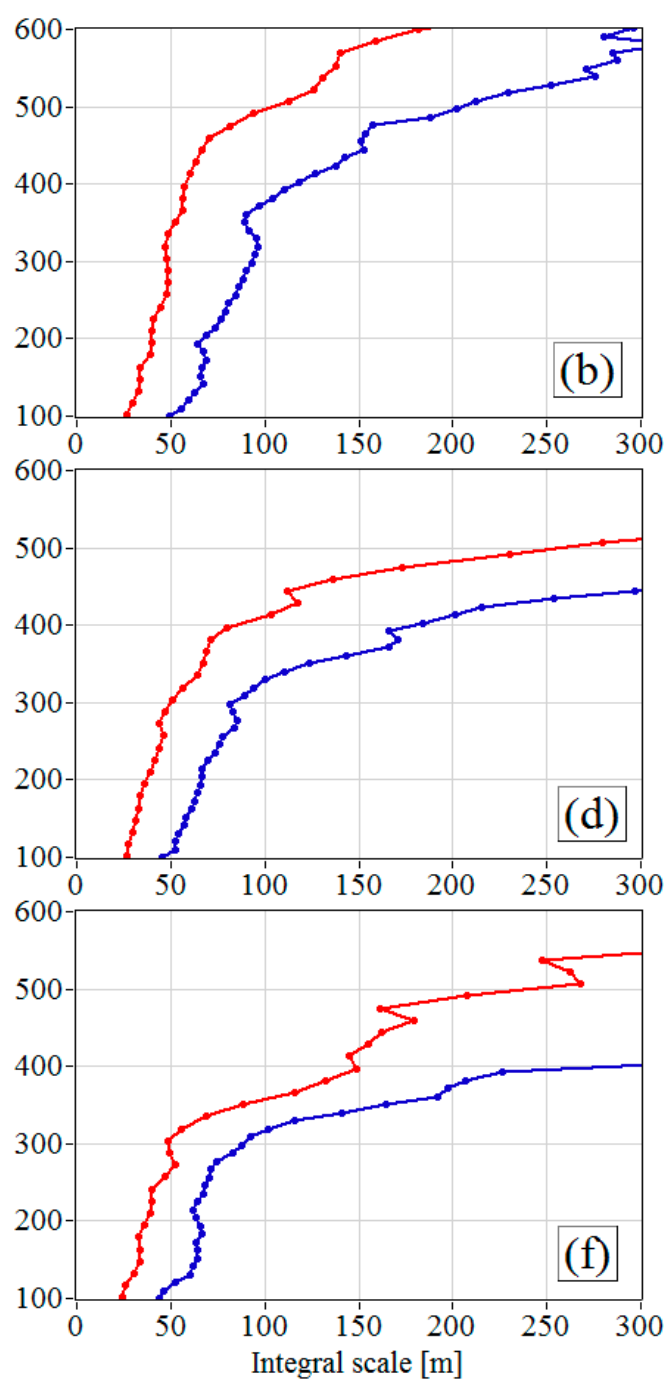

Figure 6. Vertical profiles of the $(\mathbf{a}, \mathbf{c}, \mathbf{e})$ radial velocity variance and $(\mathbf{b}, \mathbf{d}, \mathbf{f})$ integral scale of turbulence retrieved from measurements by the lidar at the elevation angles of $35.3^{\circ}$ (blue curves) and $60^{\circ}$ (red curves) at (a,b) 21:00 LT on 23 July, and (c,d) 00:00 and (e,f) 03:00 LT on 24 July 2018.

From the analysis of the results of estimation of the turbulent energy dissipation rate $\varepsilon_{i}$ shown in Figure $5, \varepsilon_{1} \approx \varepsilon_{2}$ at heights of $100-320 \mathrm{~m}$ and $\varepsilon_{1}>\varepsilon_{2}$ at heights of 320-500 m. This means that $L_{V 1}$ obtained by Equation (9) in the 320-500 m layer is underestimated, whereas the $L_{V 2}$ in this layer is overestimated. In spite of that, the inequality $L_{V 1}(h)>L_{V 2}(h)$ holds at all heights, due to the anisotropy of turbulence (Figure $6 \mathrm{~b}, \mathrm{~d}, \mathrm{f}$ ).

The data in Figure $3 a-b$ show that the ratio of the radial velocity variances $\mu=\sigma_{r 1}^{2} / \sigma_{r 2}^{2}$ averaged employing all estimates in the 100-300 $\mathrm{m}$ layer is equal to 1.4. Thus, on average, the ratio of the integral scales is $L_{V 1} / L_{V 2}=\mu^{3 / 2}=1.7$. Approximately the same result $L_{V 1} / L_{V 2} \approx 1.8$ was obtained from direct averaging of the ratios of the integral scales in this layer based on the data in Figure 3e-f. Thus, due to the anisotropy of turbulence, the variance of the radial velocity at the elevation angle of $35.3^{\circ}$ is 1.4 times larger than that at $60^{\circ}$, whereas the integral scale of turbulence at the elevation angle of $35.3^{\circ}$ exceeds the integral scale at the elevation angle of $60^{\circ}$ by 1.7 times.

Assuming that the wind field is horizontally statistically homogeneous in the 100-300 m layer, applying $\sigma_{r i}^{2}=\sigma_{w}^{2} \sin ^{2} \varphi_{i}+\sigma_{H}^{2} \cos ^{2} \varphi_{i}$ (Equation (3) [20]), where $\phi_{1}=35.3^{\circ}$ and $\phi_{2}=60^{\circ}$, we find:

$$
v_{\sigma}=\frac{\sigma_{H}^{2}}{\sigma_{w}^{2}}=\frac{9 \mu-4}{8-3 \mu}
$$


for the ratio of the average between the variances of the longitudinal and transversal components of the wind vector $\sigma_{H}^{2}=\left(\sigma_{u}^{2}+\sigma_{v}^{2}\right) / 2$ to the variance of vertical component $\sigma_{w}^{2}$. Substituting $\mu=1.4$ into Equation (10) yields that the variance $\sigma_{H}^{2}$ is 2.26 times larger than the variance $\sigma_{w}^{2}$. On the assumption that the spatial structure of the wind turbulence is described by the von Kàrmàn model (Equation (1)), we obtain $v_{L}=L_{H} / L_{w}=\left(\sigma_{H}^{2} / \sigma_{w}^{2}\right)^{3 / 2}$ for the ratio of integral longitudinal correlation scales of fluctuations in the horizontal $L_{H}$ and vertical $L_{w}$ components of the wind velocity vector. Substituting $\sigma_{H}^{2} / \sigma_{w}^{2}=2.26$ into this formula yields that the correlation scale of fluctuations of the horizontal wind component $L_{H}$ is 3.4 times larger than that of fluctuations of the vertical component $L_{w}$. The obtained estimates of the anisotropy coefficient of wind turbulence parameters do not contradict the known experimental data [28].

Using the data (three components of the wind vector) measured with a sonic anemometer at a height of $42 \mathrm{~m}$ (Figure 4), we obtained the variances $\sigma_{H}^{2}(t)$ and $\sigma_{w}^{2}(t)$ versus time $t$ for the period under consideration from 20:00 on 23 July to $08: 00$ on 24 July 2018. Each variance estimate was derived from 30-minute measurement data. The anisotropy coefficient $\sigma_{H}^{2} / \sigma_{w}^{2}$ averaged over all the measurement period was approximately 2 , which is only $13 \%$ less than the lidar estimate $\left(v_{\sigma}=2.26\right)$ for heights of 100-300 m.

As follows from Figures $3 \mathrm{e}-\mathrm{f}$ and $6 \mathrm{~b}, \mathrm{~d}, \mathrm{f}$, the lidar estimate of the integral scale $L_{V i}$ increases with height and takes overstated values at heights above $450 \mathrm{~m}$ at the central and upper parts of the LLJ. In Figure $3 \mathrm{e}-\mathrm{f}$, the zones where the estimates of the integral scale of turbulence $L_{V i}\left(h_{k i}, t_{n}\right)$ exceed 200 $\mathrm{m}$ are shown in white. To find the reason for this error, by analogy with Banakh and Smalikho. [25], we studied fluctuations in the lidar estimates of the radial velocity $V_{L i}^{\prime}\left(R_{k}, \theta_{m} ; n\right)$. As a result, we found that inhomogeneities in the wind flow with scales comparable to the base radius of the scan cone occur above $300 \mathrm{~m}$. This effect is mostly pronounced at the central part of the LLJ and especially in the zones where the wind direction changes strongly with height (Figures $2 \mathrm{e}-\mathrm{f}$ and $4 \mathrm{~b}$ ). It is possible that mesoscale processes occur here, like internal atmospheric waves arising under stable temperature stratification conditions and a strong change in the wind velocity with height.

For a more accurate estimation of the scales $L_{H}$ and $L_{w}$ in the upper part of the LLJ, we used the following approach. Consider the 100-300 m layer, where the contribution of mesoscale processes to wind variations is negligibly small in comparison with wind turbulence, and the conditions of horizontally statistically homogeneous turbulence are fulfilled $\left(\varepsilon_{1} \approx \varepsilon_{2}\right.$, Figure 5$)$. In this layer, as depicted in Figure $6 \mathrm{~b}, \mathrm{~d}, \mathrm{f}$, on average, the integral scales $L_{V 1}$ and $L_{V 2}$ increase linearly with height and have realistic values. Thus, at a height $100 \mathrm{~m}$, on average, $L_{V 1}=45 \mathrm{~m}$ and $L_{V 2}=26 \mathrm{~m}$, whereas at $300 \mathrm{~m} L_{V 1}=93 \mathrm{~m}$ and $L_{V 2}=51 \mathrm{~m}$. Assuming that in the 300-500 m layer, the characteristic of the change in the integral scale $L_{V i}$ with height does not considerably differ from that observed in the lower layer, we linearly extrapolated the scales in the 100-300 m layer to higher layers. As a result, we found that at $h=500 \mathrm{~m}$, the integral scales are $L_{V 1}=136 \mathrm{~m}$ and $L_{V 2}=80 \mathrm{~m}$. These values of the turbulent scales $L_{V i}$ for the central part of the LLJ do not contradict the published experimental data [25]. According to Equations (7) and (8), at the central part of the LLJ at a height of $500 \mathrm{~m}$ with $L_{V 1}=136 \mathrm{~m}$ and $L_{V 2}=80 \mathrm{~m}$, the horizontal $L_{H}$ and vertical $L_{w}$ scales of longitudinal correlation of wind velocity are approximately $183 \mathrm{~m}$ and $54 \mathrm{~m}$, respectively.

\section{Conclusions}

In this paper, we proposed a method for the determination of wind turbulence parameters from PCDL measurements employing conical scanning at two different elevation angles. This method assesses the anisotropy of the spatial correlation of the wind velocity turbulent fluctuations. The method was successfully tested in an atmospheric experiment with a Stream Line lidar (Halo Photonics, Brockamin, Worcester, United Kingdom) under conditions of stable temperature stratification of the atmospheric boundary layer.

We found that the variance in radial velocity measured at an elevation angle of $35.3^{\circ}$ always exceeds the variance in radial velocity measured at an elevation angle of $60^{\circ}$. The averaged ratio of 
these variances is 1.4. Assuming that the turbulence spatial spectrum is described by the von Kàrmàn model, we obtained that the ratio of the integral scales of fluctuations of the radial velocity measured with scanning at angles of $35.3^{\circ}$ and $60^{\circ}$ is approximately equal to 1.7 . It follows from the obtained estimates of turbulence anisotropy that the variance of the horizontal velocity exceeds the variance of the vertical wind velocity 2.26 times. Correspondingly, the ratio of the integral correlation scale of fluctuations of the horizontal wind velocity to that of the vertical velocity is approximately 3.4. At the central part of the LLJ observed during the experiment, at heights of 400-500 m, the integral scales of fluctuations of the horizontal and vertical wind velocity components are, on average, 183 and $54 \mathrm{~m}$, respectively.

Author Contributions: Conceptualization, V.A.B.; Methodology, V.A.B. and I.N.S.; Software, I.N.S.; Validation, V.A.B. and I.N.S.; Investigation, V.A.B. and I.N.S.; Writing-Original Draft Preparation, I.N.S.; Writing-Review \& Editing, V.A.B.; Visualization, I.N.S.; Supervision, V.A.B.; Project Administration, V.A.B.; Funding Acquisition, V.A.B.

Funding: The work was performed under the Russian Science Foundation project 19-17-00170.

Acknowledgments: The authors are grateful to our colleagues A.V. Falits and A.M. Sherstobitov for their help in conducting the lidar experiment and processing the experimental data.

Conflicts of Interest: The authors declare no conflict of interest. The funders had no role in the design of the study; in the collection, analyses, or interpretation of data; in the writing of the manuscript, and in the decision to publish the results.

\section{References}

1. Barenblatt, G.I.; Monin, A.S. On a possible mechanism of the phenomenon of discoid formations in the atmosphere. Dokl. Akad. Nauk SSSR 1979, 246, 834-837. (In Russian)

2. Gurvich, A.S.; Brekhovskikh, V.L. Study of the Turbulence and Inner Waves in the Stratosphere Based on the Observations of Stellar Scintillations from Space: A Model of Scintillation Spectra. Waves Random Media 2001, 11, 163-181. [CrossRef]

3. Gurvich, A.S.; Kan, V. Structure of air density irregularities in the stratosphere from spacecraft observations of stellar scintillation: 1 . Three-dimensional spectrum model and recovery of its parameters. 2. Characteristic scales, structure characteristics, and kinetic energy dissipation. Izv. Atmos. Ocean. Phys. 2003, 39, 300-321.

4. Kan, V.; Gorbunov, M.E.; Sofieva, V.F. Fluctuations of radio occultation signals in sounding the Earth's atmosphere. Atmos. Meas. Tech. 2018, 11, 663-680. [CrossRef]

5. Blackadar, A.K. Boundary layer wind maxima and their significance for the growth of nocturnal inversions. Bull. Am. Meteorol. Soc. 1957, 38, 283-290. [CrossRef]

6. Eberhard, W.L.; Cupp, R.E.; Healy, K.R. Doppler lidar measurement of profiles of turbulence and momentum flux. J. Atmos. Ocean. Technol. 1989, 6, 809-819. [CrossRef]

7. Frehlich, R.G.; Hannon, S.M.; Henderson, S.W. Coherent Doppler lidar measurements of wind field statistics. Bound.-Layer Meteorol. 1998, 86, 223-256. [CrossRef]

8. Smalikho, I.N.; Köpp, F.; Rahm, S. Measurement of atmospheric turbulence by 2- $\mu$ m Doppler lidar. J. Atmos. Ocean. Technol. 2005, 22, 11, 1733-1747. [CrossRef]

9. Frehlich, R.G.; Meillier, Y.; Jensen, M.L.; Balsley, B.; Sharman, R. Measurements of boundary layer profiles in urban environment. J. Appl. Meteorol. Climatol. 2006, 45, 821-837. [CrossRef]

10. Banta, R.M.; Pichugina, Y.L.; Brewer, W.A. Turbulent velocity-variance profiles in the stable boundary layer generated by a nocturnal low-level jet. J. Atmos. Sci. 2006, 63, 2700-2719. [CrossRef]

11. Banakh, V.A.; Smalikho, I.N.; Pichugina, E.L.; Brewer, W.A. Representativeness of Measurements of the Dissipation Rate of Turbulence Energy by Scanning Doppler Lidar. Atmos. Ocean. Opt. 2010, 23, 48-54. [CrossRef]

12. O'Connor, E.J.; Illingworth, A.J.; Brooks, I.M.; Westbrook, C.D.; Hogan, R.J.; Davies, F.; Brooks, B.J. A method for estimating the kinetic energy dissipation rate from a vertically pointing Doppler lidar, and independent evaluation from balloon-borne in situ measurements. J. Atmos. Ocean. Technol. 2010, 27, 1652-1664. [CrossRef] 
13. Sathe, A.; Mann, J. A review of turbulence measurements using ground-based wind lidars. Atmos. Meas. Tech. 2013, 6, 3147-3167. [CrossRef]

14. Banakh, V.A.; Smalikho, I.N. Coherent Doppler Wind Lidars in a Turbulent Atmosphere; Artech House Publishers: Boston, MA, USA; London, UK, 2013; ISBN 978-1-60807-667-3.

15. Sathe, A.; Mann, J.; Vasiljevic, N.; Lea, G. A six-beam method to measure turbulence statistics using ground-based wind lidars. Atmos. Meas. Tech. 2014, 7, 10327-10359. [CrossRef]

16. Fuertes, F.C.; Iungo, G.V.; Porté-Agel, F. 3D turbulence measurements using three synchronous wind lidars: Validation against sonic anemometry. J. Atmos. Ocean. Technol. 2014, 31, 1549-1556. [CrossRef]

17. Smalikho, I.N.; Banakh, V.A.; Falits, A.V.; Rudi, Y.A. Determination of the turbulent energy dissipation rate from data measured by a "Stream Line" lidar in the atmospheric surface layer. Opt. Atmos. Okeana 2015, 28, 901-905. (In Russian) [CrossRef]

18. Newman, J.F.; Klein, P.M.; Wharton, S.; Sathe, A.; Bonin, T.A.; Chilson, P.B.; Muschinski, A. Evaluation of three lidar scanning strategies for turbulence measurements. Atmos Meas. Tech. 2016, 9, 1993-2013. [CrossRef]

19. Smalikho, I.N.; Banakh, V.A.; Falits, A.V. Lidar measurements of wind turbulence parameters in the atmospheric boundary layer. Opt. Atmos. Okeana 2017, 30, 342-349. (In Russian) [CrossRef]

20. Smalikho, I.N.; Banakh, V.A. Measurements of wind turbulence parameters by a conically scanning coherent Doppler lidar in the atmospheric boundar layer. Atmos. Meas. Tech. 2017, 10, 4191-4208. [CrossRef]

21. Bonin, T.A.; Choukulkar, A.; Brewer, W.A.; Sandberg, S.P.; Weickmann, A.M.; Pichugina, Y.; Banta, R.M.; Oncley, S.P.; Wolfe, D.E. Evaluation of Turbulence Measurement Techniques from a Single Doppler Lidar. Atmos. Meas. Tech. 2017, 10, 3021-3039. [CrossRef]

22. Newman, J.F.; Clifton, A. An error reduction algorithm to improve lidar turbulence estimates for wind energy. Wind Energy Sci. 2017, 2, 77-95. [CrossRef]

23. Bodini, N.; Lundquist, J.K.; Newsom, R.K. Estimation of turbulence dissipation rate and its variability from sonic anemometer and wind Doppler lidar during the XPIA field campaign. Atmos. Meas. Tech. 2018, 11, 4291-4308. [CrossRef]

24. Stephan, A.; Wildmann, N.; Smalikho, I.N. Measurements of wind turbulence parameters by a Windcube 200s lidar in the atmospheric boundary layer. Opt. Atmos. Okeana 2018, 31, 815-820. (In Russian) [CrossRef]

25. Banakh, V.A.; Smalikho, I.N. Lidar studies of wind turbulence in the stable atmospheric boundary layer. Remote Sens. 2018, 10, 1219. [CrossRef]

26. Von Kàrmàn, T. Progress in the statistical theory of turbulence. Proc. Natl. Acad. Sci. USA 1948, 34, 530-539. [CrossRef] [PubMed]

27. Smalikho, I.N.; Banakh, V.A. Accuracy of Estimation of the Turbulent Energy Dissipation Rate from Wind Measurements with a Conically Scanning Pulsed Coherent Doppler Lidar. Part I. Algorithm of Data Processing. Atmos. Ocean. Opt. 2013, 26, 404-410. [CrossRef]

28. Byzova, N.L.; Ivanov, V.N.; Garger, E.K. Turbulence in Atmospheric Boundary Layer; Gidrometeoizdat: Leningrad, Russia, 1989; p. 265, (In Russian). ISBN 5-286-00151-3.

29. Smalikho, I. Techniques of wind vector estimation from data measured with a scanning coherent Doppler lidar. J. Atmos. Ocean. Technol. 2003, 20, 276-291. [CrossRef]

30. Banakh, V.A.; Smalikho, I.N.; Falits, V.A. Estimation of the turbulence energy dissipation rate in the atmospheric boundary layer from measurements of the radial wind velocity by micropulse coherent Doppler lidar. Opt. Express 2017, 25, 22679-22692. [CrossRef] [PubMed]

31. Lolli, S.; Delaval, A.; Loth, C.; Garnier, A.; Flamant, P.H. 0.355-micrometer direct detection wind lidar under testing during a field campaign in consideration of ESA's ADM-Aeolus mission. Atmos. Meas. Tech. 2013, 6, 3349-3358. [CrossRef]

32. Frehlich, R.G.; Yadlowsky, M.J. Performance of mean-frequency estimators for Doppler radar and lidar. J. Atmos. Ocean. Technol. 1994, 11, 1217-1230. [CrossRef]

(C) 2019 by the authors. Licensee MDPI, Basel, Switzerland. This article is an open access article distributed under the terms and conditions of the Creative Commons Attribution (CC BY) license (http://creativecommons.org/licenses/by/4.0/). 